\title{
Tsallis and Levy statistics in the preparation of an earthquake
}

\author{
Y. F. Contoyiannis ${ }^{1}$ and K. Eftaxias $^{2}$ \\ ${ }^{1}$ Department of Physics and Chemistry, Techological Education Institute (TEI) of Chalkis, Greece \\ ${ }^{2}$ Department of Physics, University of Athens, 15771 Athens, Greece
}

Received: 21 December 2007 - Revised: 19 March 2008 - Accepted: 3 April 2008 - Published: 6 May 2008

\begin{abstract}
Precursory fracture induced electromagnetic (EM) emissions, rooted in opening cracks and ranging from $\mathrm{MHz}$ to $\mathrm{kHz}$, with the $\mathrm{MHz}$ appearing earlier, are produced and detected both at laboratory and geophysical scale. Recently, we have proposed the following two epochs/stages model of EQ generation: (i) The final $\mathrm{kHz}$ part is triggered by the fracture of high strength and large asperities that are distributed along the activated fault and sustain the system. (ii) The initial $\mathrm{MHz}$ part is thought to be due to the fracture of highly heterogeneous system that surrounds the family of asperities. Interestingly, the MHz EM time-series can be described in analogy with a thermal second order phase transition. Herein we focus on the MHz pre-seismic activity, and especially on the naturally arising question: what is the physical mechanism that organizes the heterogeneous system in its critical state? Combining ideas of Levy and Tsallis statistics and criticality with features hidden in the precursory $\mathrm{MHz}$ time-series we argue that a Levy walk type mechanism can organize the heterogeneous system to criticality. Based on a numerically produced truncated Levy walk, we propose a way to estimate in the stage of critical fluctuations: (i) the associated Levy index- $a$, which describes quantitatively the underlying Levy dynamics, and (ii) the range of values where the nonextesitive Tsallis index $q$ is restricted. We also show that the $\mathrm{kHz}$ EM activity could not be described by a truncated Levy mechanism. This result further indicates an abrupt sweep of the population of asperities that sustain the system.
\end{abstract}

\section{Introduction}

Earthquakes (EQs) are large-scale fracture phenomena in the Earth's heterogeneous crust. Despite the large amount of experimental data and the considerable effort that has been undertaken by the material scientists, many questions about

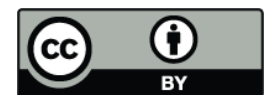

Correspondence to: K. Eftaxias (ceftax@phys.uoa.gr) fracture processes remain standing. Especially, many aspects of EQ generation still escape our full understudying.

Fracture induced physical fields allow a real-time monitoring of damage evolution in materials during mechanical loading. Electromagnetic (EM) emissions in a wide frequency spectrum ranging from $\mathrm{kHz}$ to $\mathrm{MHz}$ are produced by opening cracks, which can be considered as the so-called precursors of general fracture. These precursors are detectable both at a laboratory and a geophysical scale. It is by now recognized that the pre-fracture $\mathrm{kHz}-\mathrm{MHz} \mathrm{EM}$ time series contain valuable information about the fracture EQ preparation process (Kapiris et al., 2004; Karamanos et al., 2005, 2006). Being non-destructive, monitoring techniques based on these fracture-induced fields are a basis for a fundamental understanding of fracture mechanism and for development consecutive models of rock/focal area behavior.

Our main observational tool is the monitoring of the fracture which occurs in the focal area before the final break-up by recording their kHz-MHz EM emissions. Clear kHz-to$\mathrm{MHz}$ EM anomalies have been detected over periods ranging from a few days to a few hours prior to recent destructive EQs in Greece. We emphasize that the $\mathrm{MHz}$ radiation appears earlier than the $\mathrm{kHz}$ (Contoyiannis et al., 2005). This situation is in harmony with laboratory results.

An important challenge in this field of research is to distinguish characteristic epochs in the evolution of precursory EM activity and identify them with the equivalent last stages in the EQ preparation process. In a recent work (Contoyiannis et al., 2005), we approach the preparation of EQs in terms of critical phase transitions. Based on this analysis we have proposed the following two epochs/stages model of EQ generation (i) The final $\mathrm{kHz}$ part is triggered by the fracture of high strength, large, and rather homogeneous asperities that are distributed along the activated fault sustaining the system. (ii) The initial MHz part is thought to be due to the fracture of highly heterogeneous system that surrounds the family of asperities. Importantly, the MHz epoch is described in analogy with a thermal continuous phase transition. A fractal spectral analysis by means of Hurst exponent shows that this activity

Published by Copernicus Publications on behalf of the European Geosciences Union and the American Geophysical Union. 
has anti-persistent properties, i.e., if the EM fluctuations increase in a period, they are likely to continue decreasing in the interval immediately following and vice versa. An antipersistent behavior (Contoyiannis et al., 2005; Karamanos et al., 2006) implies a set of fluctuations tending to induce a stability within the system, i.e., a nonlinear negative feedback in the underlying fracto-EM mechanism, which "kicks" the opening cracks away from extremes. The observed antipersistency, which is rooted in the heterogeneity of the system, enables the fracture in highly heterogeneous systems to be described via an analogy with thermal continuous phase transitions. On the contrary, the abrupt emergence of strong impulsive $\mathrm{kHz}$ EM emission indicates a nonequilibrium process without any footprint of an equilibrium second order phase transition. Moreover, this time series shows persistent behavior, i.e., if the amplitude of EM fluctuations increases in a time interval, it is likely to continue increasing in the interval immediately following. The persistency, which may reflect the narrow distribution of strength in the backbone of asperities, implies a nonlinear positive feedback in the underlying fracto-EM mechanism. The existence of a positive feedback justifies the absent any signature of a second order transition. In fracture mechanics, the democratic fiber bundle model (DFBM) exhibits an interesting transition as a function of the amplitude of the disorder. There exists a tricritical transition from a Griffith-type abrupt rupture, i.e., "firstorder" regime, where rupture occurs without significant precursors, to a progressive damage, i.e., "second-order" regime as the disorder increases. We argue that the observed features in the precursory EM emissions are in agreement with this scheme.

The above mentioned proposal, due to its crucial character, needs further justification. Recently, based on "scale invariance" and "universality" concepts we have supported the hypothesis that the $\mathrm{kHz}$ EM activity is originated during the fracture of asperities (Contoyiannis et al., 2005). In this work, we focus on the first epoch/stage of the EQ nucleation process, namely, on the fracture of the heterogeneous component of the focal area. A question naturally arises refers to the physical mechanism that organizes a heterogeneous system in its critical state. We attempt to established the hypothesis that a Levy walk type fracture mechanism could be a candidate relevant critical mechanism. Physicists working in statistical physics started to suggest in the mid-eighties that rupture of sufficiently heterogeneous media would exhibits universal properties, in a way maybe similar to critical transitions (Sornette et al., 2004). However, the fracture process, especially when energies are high enough, leads to the emergence of long-range correlations between all parts of the area being fractured. This situation violates the BoltzmannGibbs statistics, the bridge to the equilibrium thermodynamics. Then, the use of a nonadditive statistics, such as the Tsallis statistics, seems to be adequate. Importantly, the Levy statistics has been quantitatively connected with the nonadditive Tsallis statistics. Herein, based on this connection, we try to extract more information possibly hidden in the $\mathrm{MHz}$ EM time series about the mechanism that organizes the critical stage of fracture.

This paper is organized as following: In Sect. 2 we briefly present the foundations of the recently introduced method of critical fluctuations (MCF). Based on this method, we investigate the preseismic EM time series in terms of criticality. In Sect. 3 we refer to a connection between the Levy statistics and Tsallis statistics, as it has been presented in literature. In Sect. 4 a relation between characteristic indexes of the MCF and the Levy statistics is deduced. In Sect. 5, based on a Monte-Carlo algorithm, we produce a two-dimensional Levy walk portrait. We investigate whether for an appropriate set of the associated parameters the Levy walk would exhibit critical signatures in the associated time series. In Sect. 6 we present the application of the MCF on the preseismic MHz EM time series associated with the Athens EQ. In Sect. 7, a proposed scenario seems to justify that a Levy walk type fracture mechanism can organize the criticality in the heterogeneous environment. In Sect. 8, an estimation of Tsallis q-index associated with the fracture in heterogeneous environment is accomplished. Finally, in Sect. 9, we present the conclusions of this work.

\section{The method of critical fluctuations}

In Contoyiannis and Diakonos (2000) we investigated how a thermal system at its critical point can be described in terms of dynamical systems. We found that the critical fluctuations of the order parameter $\phi$ obey to a dynamical law which is described by the following intermittent map

$\phi_{n+1}=\phi_{n}+u \phi_{n}^{z}+\epsilon_{n}$

where $\epsilon_{n}$ is a shift parameter.

All the parameters of this map have a clear physical content. So, the exponent $\mathrm{z}$ is connected with the isothermal critical exponent $\delta$ as $z=\delta+1$. Each physical system has its characteristic "noise", which is expressed through the shift parameter $\epsilon_{n}$. The critical state is characterized by the unstable fixed point. For example, for a magnetic phase transition the unstable fixed point has the value zero (mean magnetization). Importantly, the statistical properties of distribution of laminar lengths, namely the distribution of stay times, in the neighbourhood of the unstable fixed point associated with the critical state, are similar for various systems. This evidence leads to the same exponent $z$, because the intermittent dynamics is controlled by the nonlinear term of Eq. (1).

In Contoyiannis et al. $(2002,2004,2005)$ we have developed the Method of Critical Fluctuations (MCF), which can reveal crucial information about the critical state. The MCF is described through the statistical distribution of laminar lengths $l$ for the map (1) (Schuster, 1989):

$P(l) \sim l^{-p l}$ 
where the exponent $p_{l}$ is connected with the exponent $z$ as $p_{l}=\frac{z}{z-1}$. Therefore the exponent $p_{l}$ is connected with the isothermal exponent as:

$p_{l}=1+\frac{1}{\delta}$

Due to the universal character of critical phenomena the exponent $p_{l}$ can refer to systems beyond the thermal ones which appear similar dynamical fluctuations (Contoyiannis et al., 2002). The appearance of such fluctuations could be a signal of an underlying critical state. The MCF is directly applied to time-series or to segments of time series which appear a cumulative type stationary behaviour.

The main aim of MCF is to estimate the exponent $p_{l}$. The distribution of the laminar lengths, is fitted in the frame of the MCF by the function:

$R(s) \sim s^{-p_{2}} e^{-p_{3} s}$

We focus on the exponents $p_{2}$ and $p_{3}$. If the exponent $p_{3}$ is zero, then, the exponent $p_{2}$ is equal to the exponent $p_{l}$. Practically, as the exponent $p_{3}$ approaches to zero, then, the exponent $p_{2}$ approaches to $p_{l}$, while, the laminar lengths tend to follow a power-law type distribution. We note that Eq. (3) suggests that the exponent $p_{l}$ (or $p_{2}$ ) should be greater than 1.

In summary, the condition of criticality has been found as $p_{2}>1$ and $p_{3} \approx 0$ (Contoyiannis et al., 2002, 2004, 2005). We stress that when the exponent $p_{2}$ is smaller than one, then, independently of the $p_{3}$-value, the system is not at a critical state. Generally, the exponents $p_{2}, p_{3}$ have a competitive character, namely, when the exponent $p_{2}$ decreases the associated exponent $p_{3}$ increase. In this way, we can identify the deviation from the critical state. To be more concrete, as the exponent $p_{2}\left(p_{2}<1\right)$ is close to 1 and simultaneously the exponent $p_{3}$ is close to zero, then, the system is at a subcritical state. As the system removes from the critical state, then, the exponent $p_{2}$ decreases while simultaneously the exponent $p_{3}$ increases reinforcing, in this way, the exponential character of the laminar lengths distribution. A situation like this indicates that the dynamics of the system becomes rather chaotic than deterministic (Pingel and al., 1999; Diakonos and al., 1999). From all the above mentioned findings we conclude that the research of criticality in natural systems could be quantitatively accomplished by estimating the values of only two parameters, namely the exponents $p_{2}$ and $p_{3}$. Up to now, we have applied the MCF on numerical experiments of thermal systems (Ising models) (Contoyiannis et al., 2002), on electromagnetic pre-seismic signals (Contoyiannis et al., 2005), and on electrocardiac signals from biological tissues (Contoyiannis et al., 2004).

\section{$3 \quad$ Levy and Tsallis statistics}

The Levy Statistics is applied to physical systems which develop stable processes with main characteristic the infinite variance. The characteristic function of this statistics has the form (Alemany and Zanette, 1994):

$$
G(\boldsymbol{k})=\exp \left(-c|k|^{a}\right)
$$

where $0<a<2$. This characteristic function is the Fourier transform of the jump probability $p(\boldsymbol{x})$. The previous function behaves for large $|\boldsymbol{x}|$ as (Alemany and Zanette, 1994):

$p(\boldsymbol{x}) \sim|\boldsymbol{x}|^{-(1+a)}$

The random walk process, which has the above jump probability, is known as "Levy flights".

A jump needs a time interval of duration $\tau$, which is proportional to its length $|\boldsymbol{x}|$. Such as time intervals have the character of "waiting time" between successive flights. Therefore, the corresponding probability density for these time intervals is written as:

$\Psi(\tau) \sim \tau^{-(1+a)}$

The Levy statistics has been successfully used in various dynamical systems that involve long-range spatio-temporal correlations, like anomalous diffusion phenomena, fluid turbulence, financial markets and many others.

Now, we briefly refer to the foundations of Tsallis entropy. In nature, long-range spatial interactions or long-range memory effects may give rise to very interesting behaviors. Among them, one of the most intriguing arises in systems that are nonextensive (nonadditive). These systems share a very subtle property: they violate the Boltzmann-Gibbs (BG) statistics, the bridge to the equilibrium thermodynamics. Inspired by multifractals concepts, Tsallis (1988) has proposed a generalization of the BG statistical mechanics. He introduced an entropic expression characterized by an index $q$ which lead to a nonextensive statistics,

$S_{q}=k \frac{1}{q-1}\left(1-\sum_{i=1}^{W} p_{i}^{q}\right)$,

where $p_{i}$ are the probabilities associated with the microscopic configurations, $W$ is their total number, $q$ is a real number, and $k$ is Boltzmann's constant. The value of $q$ is a measure of the nonextensivity of the system: $q=1$ corresponds to the standard, extensive, B-G statistics. Indeed, using $p_{i}^{(q-1)}=e^{(q-1) \ln \left(p_{i}\right)} \sim 1+(q-1) \ln \left(p_{i}\right)$ in the limit $q \rightarrow 1$, we recover the usual B-G entropy

$S_{1}=-k \sum_{i=1}^{W} p_{i} \ln \left(p_{i}\right)$.

More precisely, the entropic index $q$ characterizes the degree of nonextensivity reflected in the following pseudoadditivity rule:

$S_{q}(A+B)=S_{q}(A)+S_{q}(B)+(1-q) S_{q}(A) S_{q}(B)$

where $A$ and $B$ are two independent systems in the sense that the probabilities of $A+B$ factorize into those of $A$ and 
B. The values $q>1, q=1$, or $q<1$, correspond to subextensivity, extensivity or super-extensivity, respectively.

Tsallis notes that there are at least 20 entropy functions in the literature, and he compares several in detail. Importantly, the Tsallis entropy is extensive, stable, and concave.

Recently, interesting questions and investigations aiming to the quantitative description of critical phenomena through such a nonextensive statistics have been accomplished (Baldovin and Robledo., 2002; Robledo, 2004). On the other hand, a connection between Tsallis statistics and Levy statistics has been introduced (Alemany and Zanette, 1994). Therefore, it is reasonable to attempt a connection between the criticality and the Levy statistics through the Tsallis statistics. A basic scope of this work is to try such a connection.

In Alemany and Zanette (1994), the authors, based on a proper use of Tsallis entropy

$S_{q}[p(x)]=-\frac{1-\int_{\infty}^{\infty} p(x)^{q} d x}{(1-q)}$

have connected the entropic nonextensive index $q$ with the Levy index $a$ through the relation $q=\frac{3+a}{1+a}$. On the other hand, the authors in Buiatti et al. (1999) suggest that for a dynamical system, which is described through an intermittent map of the form:

$y_{n+1}=y_{n}+w y_{n}^{m} ; w>0, m>1$

the associated entropic index $q$ and the Levy index $a$ are connected with the relation

$q=\frac{2+a}{1+a}$

Notice, in Buiatti et al. (1999) the authors distinguish between: (i) a probabilistic character of Levy processes, which leads to the results presented in Alemany and Zanette (1994) and (ii) a dynamical character of Levy processes described by the map (11), which leads to the relation (12).

The stay times in the laminar region of map (11) are the waiting times between successive exits from the laminar region. The term "waiting time" has been used with a more general character, for example, as the time intervals between earthquakes (Scafetta and West, 2005).

\section{Relation between critical fluctuations exponent $p_{2}$ and Levy index $a$}

As it is mentioned, in a recent work (Robledo, 2004) the author has proposed a connection between the Tsallis statistics and critical phenomena. They concluded that the Tsallis in$\operatorname{dex} q$ and the isothermal critical exponent $\delta$ are connected via the relation:

$q=\frac{(2 \delta+1)}{(\delta+1)}$
We recall that the dynamics of critical fluctuations is described by the map (1), which has the same form as the map (11) (except the shift parameter). Thus, we can use the relation (12) between $q$ and $a$. So, a relationship between the critical exponent $p_{l}$ (or $p_{2}$ ) and Levy index $a$ is possible. Indeed, from Eqs. (12), (13) and (3) we take that:

$p_{2}=1+a$

In the case of the exact Levy Statistics and for systems in their thermodynamical limit the statistical second moment diverges. But the real systems have finite sizes. So, the description is given through the "truncated Levy walk" which has been introduced by Stanley and Mantega (Mantegna and Stanley, 1994) as a crossover between Levy and Gaussian statistics. Koponen (1995), taking account the cut-off in great scales, introduced an exponential decay in distribution of exact Levy walk. In this approach, the distribution (6) is written as:

$\Psi(\tau) \sim \tau^{-\mu} e^{-\lambda \tau}$

The cut-off parameter is denoted by the parameter $\lambda>0$. In Eq. (15) the exponent $\mu=1+a$ has been introduced. From the restriction $0<a<2$ one takes the limits of exponent $\mu$ :

$1<\mu<3$

The condition (16) is in agreement with that of criticality $p_{2}>1$.

The mechanism of Levy walk appears in many processes in nature. However, they do not assume the existence of an underlying critical state. A question naturally arises is, under what conditions a Levy walk can describe a critical state. First, we will investigate this question through a numerical experiment.

\section{Levy walk and criticality in terms of a numerical ex- periment}

Spatial patterns of truncated Levy random walks can be produced by numerical simulations. We apply such an algorithm (Antoniou et al., 2001) which produces a random Levy walk in a two dimensions space with a controlled truncation. This algorithm provides that the components $x$ of the walk is given by: $x=b(1-g \chi)^{-1 / \mu}$. Similar relation can been written for the $y$-components. In this Monte-Carlo formula, $b$ is a coefficient, $\chi$ is a random number in the interval $(0,1), \mu$ is the corresponding Levy exponent and $g$ is a parameter which determines how much truncated is the Levy walk. For $g \rightarrow 1$ the walk is possible to approach the ideal Levy walk. An interesting question is whether the Levy dynamics rooted in this algorithm could be in harmony with a critical state.

Using various g-values, keeping the other parameters $b, \mu$ constant, i.e., $b=0.1$ and $\mu=1.3$, we found that for the value $g=0.885$ there is a segment in x-time-series (see Fig. 1), 


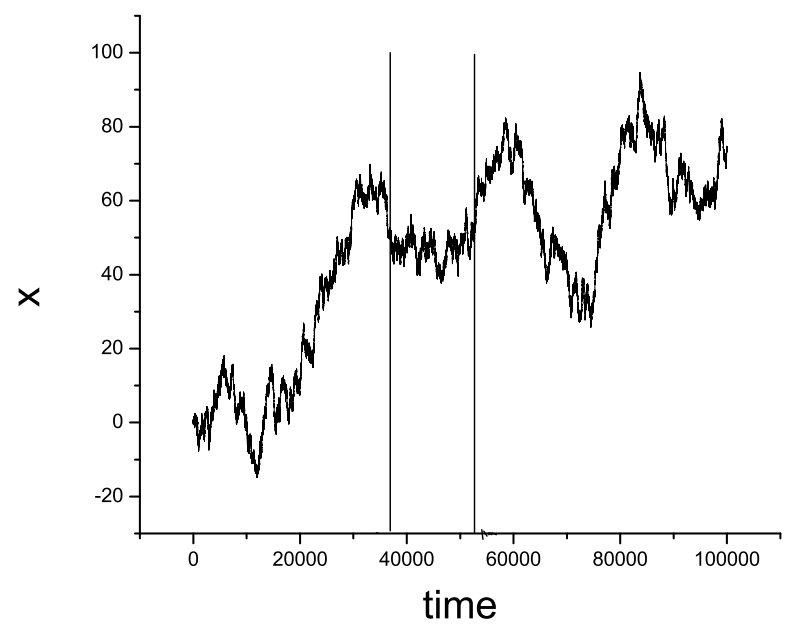

Fig. 1. A x-time-series produced by a numerical simulation associated with a truncated Levy flight random walk including 100000 steps. The time refers to the algorithmic time ( $1 \mathrm{step} / 1 \mathrm{unit})$. The stationary segment, where the analysis of criticality is accomplished, is restricted between the two vertical lines ranging from 36500 point up to 52000 point.

which has the following properties: (i) An excellent quality of cumulative stationarity, which is a necessary condition for the application of the MCF (Contoyiannis et al., 2005). In Fig. 2 this stationarity in terms of the mean values and standard deviations is presented. (ii) In Fig. 3 the distribution of laminar lengths is shown. The corresponding analysis by means of the MCF (details of this method see later) reveals that the fitting by the function (4) has the best quality $\left(r^{2}=0.988\right)$, and simultaneously, the $p_{3}$-exponent has the more close to zero value. To be more concrete, the associated exponents are $p_{2}=1.4, p_{3}=0.003$. This pair of values implies an underlying critical state. We note that the $p_{2}$-value is close enough to the corresponding Levy exponent $\mu=1.30$, as it was expected by Eq. (14). Finally, Fig. 4 demonstrates a two-dimensional portrait including a few steps.

Importantly, if we move from the above mentioned characteristic $g$-value, $g=0.885$, keeping the other parameters constant, we find that there is not any segment in x-timeseries satisfying the above mentioned properties. As an example, Fig. 5 shows the distribution of the laminar lengths for $g=0.7$. It is clear that the data can not be fitted by a power-law, and thus, any estimation of $p_{2}, p_{3}$ is impossible.

We emphasize one more that the reported agreement between criticality and Levy statistics is observed in time windows having cumulative stationary behavior. Inversely now, this agreement permits us to accomplish a quantitative estimation of the Levy index $\mu$ (or $a$ ) when the corresponding exponent of criticality $p_{2}$ has been estimated.

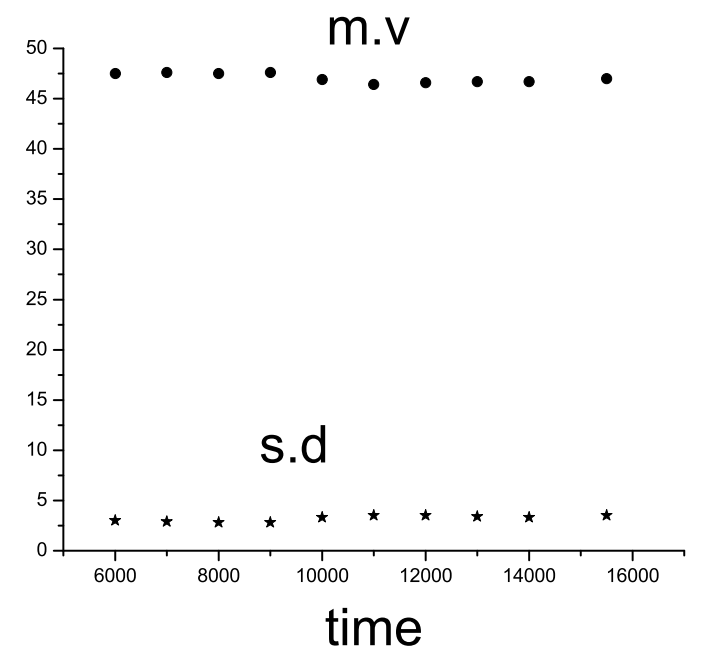

Fig. 2. The temporal evolution of cumulative mean value, as well as, that of the standard deviation, for increasing number of points, starting from an initial set of 6000 points. The plots refer to x-timeseries from a two dimensional Levy walk produced by a numerical simulation.

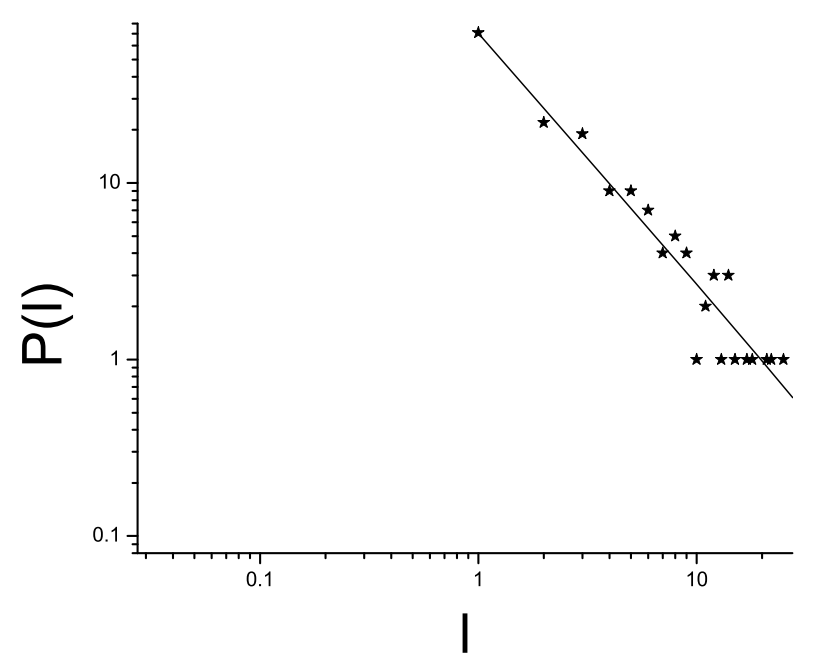

Fig. 3. The distribution of the laminar lengths from analysis in terms of criticality for the stationary segment of time series shown in Fig. 2. The solid line represents the fit of data by the fitting function (Eq. 4).

\section{Criticality in MHZ EM pre-seismic emission}

We have paid attention to the fact that such stationary windows, which also demonstrate critical fluctuations, have been found in the pre-seismic MHz EM emission associated with the Athens EQ (see Fig. 6, Contoyiannis et al., 2005). We recall, ample experimental evidence bridges this EM signal with the fracture of the strongly heterogeneous medium that surrounds the family of strong and large asperities in the focal area, as well as that this EM activity can be described in 


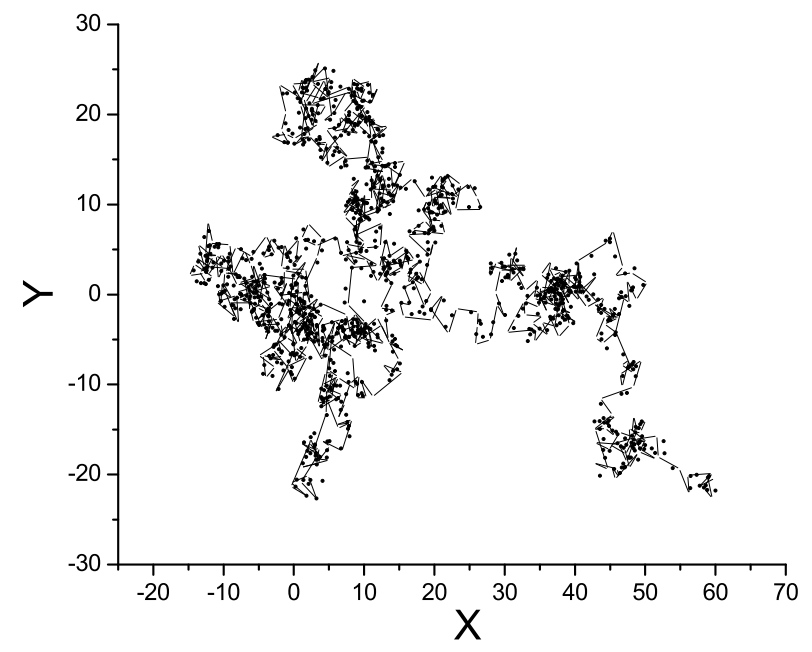

Fig. 4. A two-dimensions portrait including 30000 steps, produced from a numerical simulated two components truncated Levy flight random walk with quite small cut-off (almost 6 in arbitary units).

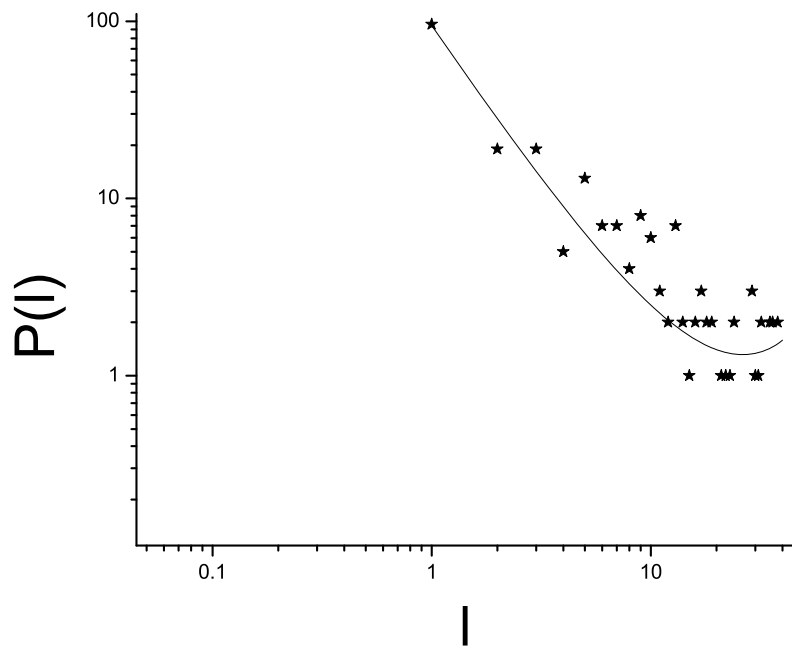

Fig. 5. The distribution of the laminar lengths, for the segment between 36500 point and 52000 points, from analysis in terms of criticality for $g=0.7, b=0.1, \mu=1.3$. It is obvious the destruction of criticality as we are removing from $g=0.885$, keeping $b$ and $\mu$ constants.

analogy with a thermal second order phase transition (Contoyiannis et al., 2005). Herein, we attempt to establish the hypothesis that the critical stage of fracture process in heterogeneous media can follow a Levy mechanism, and thus, a quantitative description of this dynamics, through the estimation of the Levy index-a, it is possible.

We focus on the two candidate precursory EM bursts of long duration I, II included in the pre-seismic EM MHz time series (see Fig. 6). Their long duration permits a valuable statistical analysis. First, we present the application of the MCF in the burst II including 65000 points. We search in this

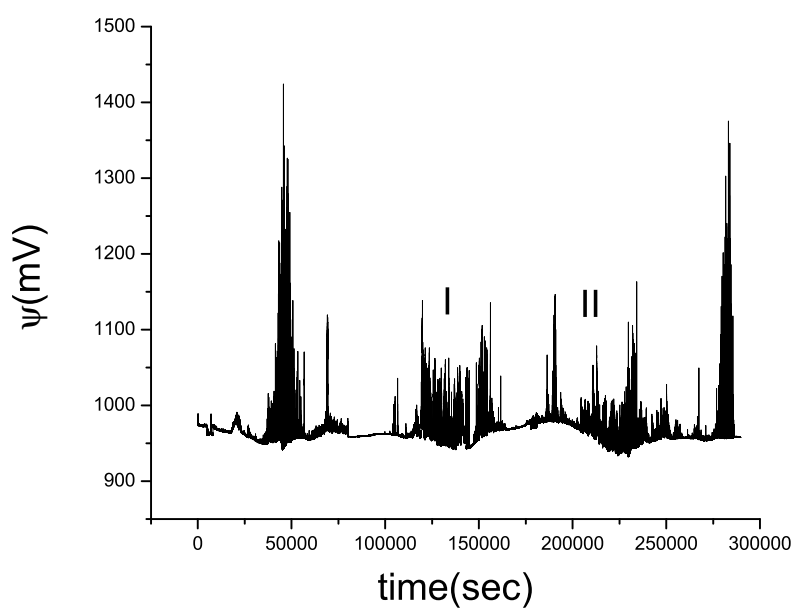

Fig. 6. The MHz EM time series recorded prior to the Athens EQ. The burst I has 52000 points and the burst II has 65000 points. The data have been recorded with sampling rate of one sample/s.

burst for a segment which shows an excellent cumulative stationary, as that observed in the case of the above mentioned numerical experiment. Indeed, we found such a stationary window with a length of $15000 \mathrm{~s}$ (see Fig. 7). The distribution $S(\psi)$ of the associated EM fluctuations $\psi$ is shown in Fig. 8. Now, we investigate the possible existence of critical fluctuations that obey the MCF.

The laminar lengths are described by the lengths of the sub-sequences in the EM time series that are resulted from successive $\psi$-values obeying the condition $\psi_{o} \leq \psi \leq \psi_{l}$, where $\psi_{o}, \psi_{l}$ are the beginning and the end of the laminar region, correspondingly. The beginning of the plateau $\left(\psi_{o} \approx 955\right)$ is the beginning of the laminar region (see Fig. 8). The end of the laminar region corresponds to the $\psi$-value which leads to the best approach of exponent $p_{3}$ to zero (Contoyiannis et al., 2002). In this way, using various exits, we find $\psi_{l}=965$. In Fig. 9 the distribution of the laminar lengths, as well as, the fitting of the data by function (4) are shown. We found that $p_{2}=1.30$ and $p_{3}=0.0077$. Consequently, the conditions that imply the existence of an underlying criticality are satisfied. For completeness, we briefly present relevant results from the EM burst I, as these have been reported in Contoyiannis et al. (2005). The window of critical fluctuations has length 20000 s (Fig. 8 in Contoyiannis et al., 2005) and the stationary condition is satisfied very well (Fig. 7 in Contoyiannis et al., 2005). The laminar distribution is shown in Fig. 9 (in Contoyiannis et al., 2005), while the exponents have the values $p_{2}=1.31$ and $p_{3}=0.0054$. It is very important to observe the similarity in the value of the critical exponent $p_{2}$, namely, $p_{2} \approx 1.3$ for the two cases studied. We recall that in terms of Levy statistics the distribution of laminar lengths is read as the distribution of waiting times which is characterized by the exponent $a=p_{2}-1$ (Eq. 14). Therefore, the critical windows in the first and 


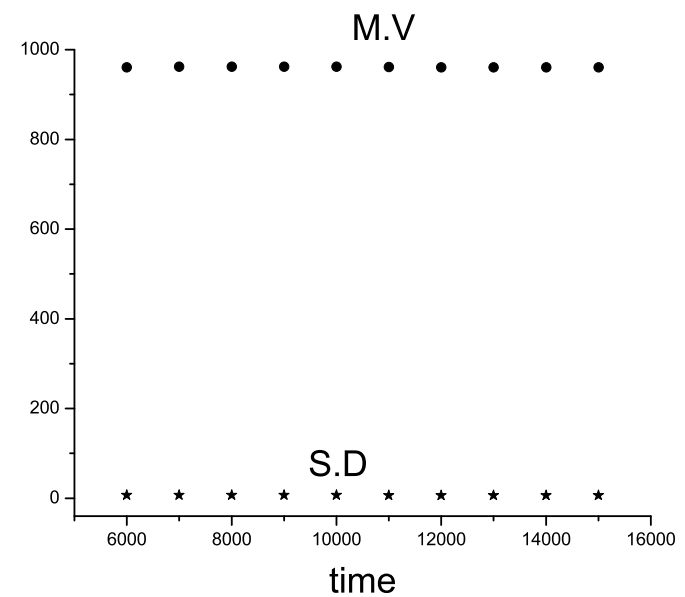

Fig. 7. The plots refer to a stationary window (15000 points) including in the EM Burst II (see Fig. 6). The figure shows temporal evolution of cumulative mean value, as well as, that of the standard deviation, for increasing number of points, starting from an initial set of 6000 points.

the second EM burst correspondingly "reflect" a Levy type walk organization of cracking network with the same exponent $\mu=1+a \approx 1.30$.

\section{A possible scenario: the organization of critical state in pre-fracture process through a Levy walk mecha- nism}

In analogy to the previously presented numerical experiment we propose a possible scenario that justifies the appearance of a Levy type mechanism in the fracture of a heterogeneous system. We consider that an "fracto-EM event" is emerged when the $\psi$-values exceed the laminar region. The time interval, during which the $\psi$-values are continually outside of the laminar region, denotes the duration (life-time) of the associated EM pulse event. Responsible for this EM emission is the cracking in a local section of the heterogeneous focal area. Failure nucleation begins to occur at a region where the resistance to rupture growth has the minimum value. The fracture continuous in the same weak region until a much stronger region is encountered in its neighbourhood. When this happens, crack growth stops. Then, in the level of EM emission, the $\psi$-values come back in the laminar region. The stresses are redistributed while the applied stress in the focal area increases, thus a new population of cracks nucleates in the weaker of the unbroken regions. The required time for the initiation of the new fracture is the waiting time. The growth of new population of cracks also stops when it encounters another stronger region, and so on. We stress that the antipersistent behavior of the EM time-series (Contoyiannis et al., 2005), supports such a scenario. In Fig. 10, the associated walk in focal area, according to the above mentioned

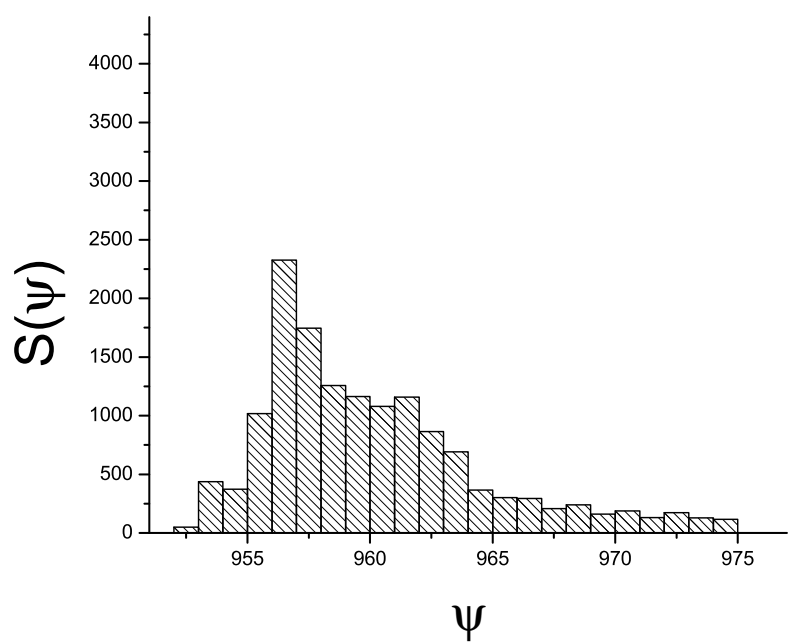

Fig. 8. The distribution $S(\psi)$ of $\psi$-values for the stationary window including in the burst II. We observe the existence of a plateau which is restricted from $\psi \approx 955$ up to $\psi \approx 962$.

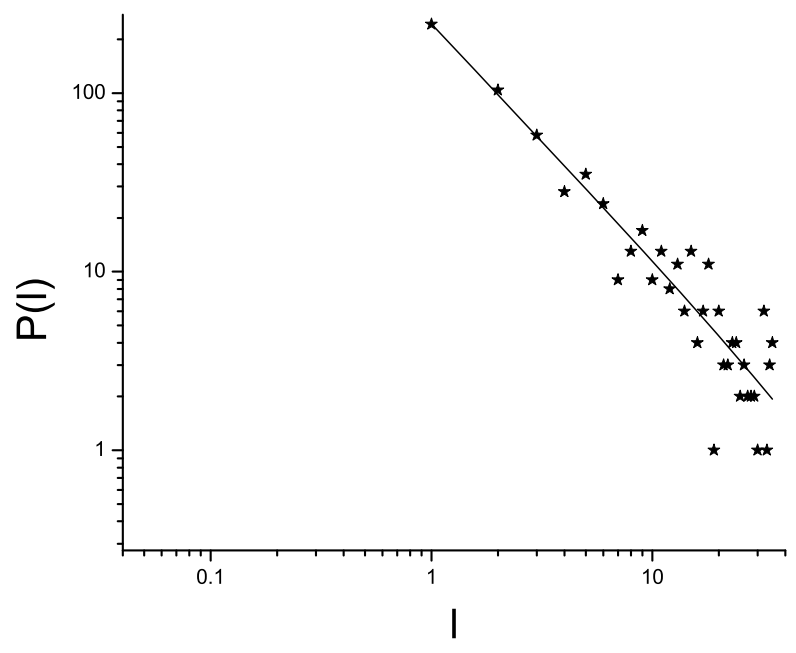

Fig. 9. The distribution of the laminar lengths, as it is produced by the analysis in terms of criticality for the stationary window including in the burst II. The fitting function (Eq. 4) is plotted with the solid line.

scenario is presented. In this figure the broken regions, as well as, the arrows representing the successive steps of the fracture in the heterogeneous environment are shown.

\section{An estimation of Tsallis index $q$ for the case of frac- ture process in heterogeneous environment}

Now we focus on the description in terms of Tsallis Statistics. From Eqs. (12) and (14) we take that:

$q=1+\frac{1}{p_{2}}$ 


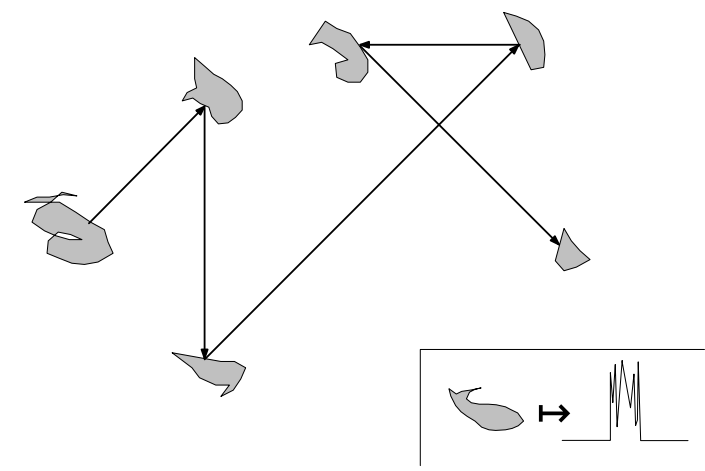

Fig. 10. A two dimensional Levy type walk according to the scenario for the fracture in a heterogenous environment (see text). The inset figure shows the EM emission which is emerged during the cracking of a local section.

As it is said, the existence of an underlying criticality imposes that $p_{2}>1$. Thus, as it results from Eq. (17), the upper limit from the $q$-index is $q<2$. Now, we refer to the lower limit of $q$-parameter that is rooted in a critical state. The fracture of a heterogeneous material is characterized by antipersistency. As it is mentioned, this implies a set of fluctuations tending to induce a stability to the system, namely, a negative non-linear feedback mechanism that "kicks" the cracking rate away from extremes. This physical picture justifies the description of fracture in heterogeneous systems in terms of a second order phase transition in equilibrium. Quantitatively, the range $0<H<0.5$, where $H$ is the Hurst exponent, describes the anti-persistency.

In Contoyiannis et al. (2005) we have introduced a relation between Hurst exponent and $p_{2}$-exponent, which predicts that the upper limit, 0.5 , of the first exponent leads to the upper limit 1.5 for the second one, and thus to the lower limit 1.66 for the $q$-parameter as it results from Eq. (17). Therefore, we suggest that the $q$-index rooted in a stationary time window included critical fluctuations is restricted in the region $1.66<q<2$. In the concrete $\mathrm{MHz}$ EM time series associated with the Athens EQ we have found that the critical exponent is $p_{2}=1.30$. Therefore, as it results from Eq. (17) $q=1.77$. We see that the estimated $q$-value obeys the previous $q$ restriction.

As it is expected, the estimated values $q>1$ represent a subextensive system: the interactions and information transition across the activated region of the crust have been verified. Notice, the estimation for the nonextensive parameters is in full agreement with the upper limit $q<2$ obtained from several independent studies involving the Tsallis nonextensive framework (Vilar et al., 2007).

As it was mentioned, the strong impulsive $\mathrm{kHz}$ EM fluctuations emerged at the tail of the precursory EM activity have been correlated with the fracture of strong and large asperities distributed along the activated fault sustaining the system (Contoyiannis et al., 2005). The analysis reveals that there is

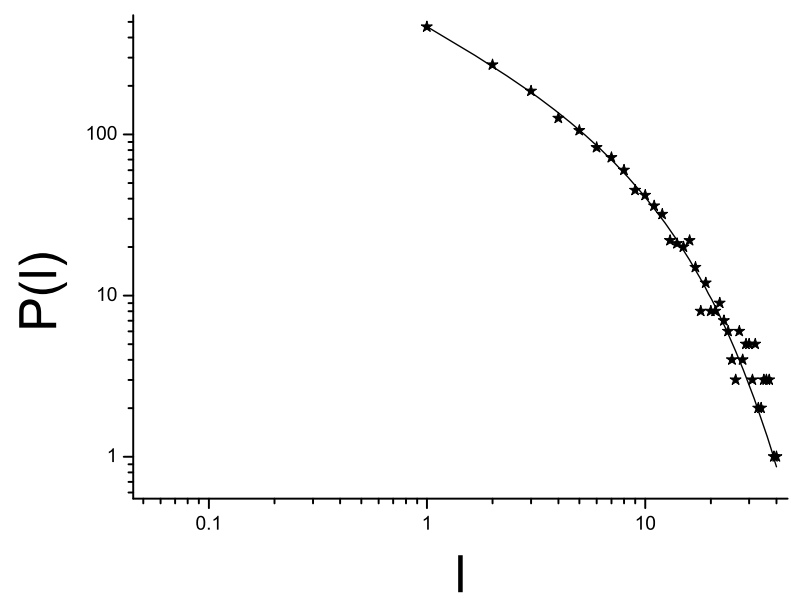

Fig. 11. A typical example of the distribution of the laminar lengths according to the analysis in terms of criticality in the a segment of $\mathrm{kHz}$ EM times series recording prior the Athens EQ. The fitting function (Eq. 4) is plotted by the solid line. The associated exponents $p_{2}, p_{3}$ indicate a transition which can not be described in terms of a second order phase transition.

not any window where the conditions of criticality in terms of MCF are valid. A typical example for such an analysis in the pre-seismic kHz EM time series is shown in Fig. 11. The associated exponents are $p_{2}=0.68$ and $p_{3}=0.1$, verifying the absence of any signature of an equilibrium phase transition. The physical feature hidden in the estimated exponents $p_{2}$, $p_{3}$ is the existence of a strong cut of the large lengths in the associated Levy walk. Importantly, this segment of EM emission shows persistency $(0.5<H<1)$ (Contoyiannis et al., 2005 ), which is consistent with a strong cut of length of consecutive steps of walk, and finally, with a abrupt "sweep" in the population of asperities.

\section{Discussion/conclusions}

EQs are large-scale fracture phenomena in the Earth's heterogeneous crust. Despite the large amount of experimental data and the considerable effort that has been undertaken by the material scientists, many questions about fracture processes remain standing. Especially, many aspects of EQ generation still escape our full understanding. Fracture induced physical EM fields, rooted in the opening cracks, allow a real-time monitoring of damage evolution in materials during mechanical loading from the laboratory up to the geophysical scale. Clear kHz-to-MHz EM anomalies have been detected over periods ranging from a few days to a few hours prior to recent destructive EQs in Greece, with the MHz radiation appearing earlier than the $\mathrm{kHz}$. Recent results indicate that these EM time-series contain information characteristic of an ensuing seismic event. A challenge in this field of research is to distinguish characteristic epochs in the evolution 
of the precursory EM activity associated with the Athens EQ and identify them with the equivalent last stages in the EQ preparation process. In this direction, our model of the focal area consists of (i) a backbone of strong and large entities distributed along the activated fault and (ii) a strongly heterogeneous medium that surrounds the family of strong entities that prevent the free slip. Based on this model, we recently proposed the following two stages model (Contoyiannis et al., 2005): The first epoch, which includes the initially emerged MHz EM activity, originates during cracking in the highly heterogeneous material that surrounds the backbone of large and strong entities. This emission could be described in analogy with a thermal continuous phase transition. The second epoch includes the $\mathrm{kHz}$ EM radiation that emerges in the tail of the precursory EM activity and ceases approximately nine hours before the Athens EQ. This activity indicates an underlying nonequilibrium process without any footprint of an equilibrium thermal phase transition. We have suggested that this radiation is due to the fracture of the backbone that sustains the system (Contoyiannis et al., 2005). Obviously, the above mentioned crucial proposal needs further documentation.

In this work we focus on the first epoch/stage. We investigate a possible mechanism which can describe the observed critical state of fracture process in the heterogeneous regime of the focal area. We have shown that a Levy walk type mechanism could be a relevant candidate mode. Based on the analysis of a numerically produced truncated Levy walk, we propose a way to estimate in the stage of critical fracto-EM fluctuations: (i) the associated Levy index- $a$, which quantitatively describes the underlying Levy dynamics; (ii) the range of values where the non-extensive Tsallis index $q$ associated with critical fluctuations is restricted; (iii) the concrete $q$ value in the windows of precursory critical EM fluctuations. Based on a proposed relation between the Tsallis index $q$ and the exponent of criticality $p_{2}$ we find that the $q$-index associated with the fracture in heterogeneous media is restricted in region $1.66<q<2$. As it is expected, the estimated values $q>1$ represent a subextensive system: the interactions and information transition across the activated region have been verified. Importantly, the estimation for this nonextensive parameter is in full agreement with the limit $q<2$ obtained from several independent studies involving the Tsallis nonextensive framework.

Intuitively, the proposed Levy walk mechanism could be the result of a feedback "dialogue" between the stresses and heterogeneity. Recently, we have introduced a self-organized model, which can describe the fracture process (Contoyiannis and Diakonos, 2006). We are going in a future work to specify this model in order to better understand the aforementioned dialogue.

A vital problem in material science and in geophysics is the identification of precursors of macroscopic defects or shocks. In physics, the degree to which we can predict a phenomenon is often measured by how well we understand it. As it is said, despite the large amount of experimental data and the considerable effort that has been undertaken by the material scientists, many questions about the fracture remain standing. This fact is reflected in the disappointing progress on short-term EQ prediction. An improved understanding of the EM precursors, especially its physical basis, has a direct implications for the EQ generation processes and EQ prediction research. The physics of EQs has been demonstrated to be a very complicated matter, and the road seems to be long and hard. A difficulty stems from the lack of large and reliable database: most studies in this area have been limited by a lack of enough experimental results to conduct a statistically significant analysis of the phenomena and obtain firm results. Another difficulty stems from the lack of collaborative efforts. We focus on these two difficulties.

It would be desirable to have the possibility to analyze more preseismic EM emissions. However, the collection of a volume of appropriate EM data for statistical purposes requires some decades of years at least. Indeed, due to their absorption these signals are associated with surface EQs of magnitude 6 or larger that occur on land or closed to coastline (Karamanos et al., 2006). However, such EQs occur rarely in Greece, so that a statistical evaluation stemming from the EM data is practically impossible (Karamanos et al., 2006). This fact obliges us to attempt a multidisciplinary evaluation of seismogenic origin of the detected candidate precursory EM emissions. Recently, a strong EQ with magnitude 6.9 occurred on 14 February (10:09:22.5 UTC) in southern Greece (36.570 N, 21.750 E) (http://www.emsc-csem.org). Precursory critical fluctuations by means of MCF have been found in the recordings of the electric antenna finely tuned at $41 \mathrm{MHz}$. These critical fluctuations lasted approximately $36 \mathrm{~h}$ and ceased at about 4 days before the EQ. The distribution of the laminar lengths (see Fig. 12) is fitted by the function (4), while the quality of fitting is excellent. The associated critical exponents are $p_{2}=1.32$ and $p_{3}=0.0072$. These values reveal a critical organization through a Levy mechanism with exponent $\mu=1.32$ and Tsallis index $q=1.76$. Importantly, these results are in full agreement with the previously presented ones associated with the Athens EQ. We note that a very clear $\mathrm{KHz}$ EM anomaly has also been detected prior to this recently occured EQ. The distribution of the laminar lengths in stationary segments of the detected anomaly is similar to that shown in Fig. 11, namely, it is characterized by an exponent $p_{2}<1$ and an exponent $p_{3}$ no close to zero. These value indicate a transition which can not be described in terms of a second order phase transition. The above mentioned findings support the proposed two stage-model scenario of the EQ nucleation in terms of precursory $\mathrm{MHz}-\mathrm{kHz}$ EM emissions (Contoyiannis et al., 2005).

In this field of research the reproducibility of the results is also desirable: the results based on $\mathrm{kHz}-\mathrm{MHz} \mathrm{EM}$ data should be verified by independent experiments. This requirement is well-satisfied in the case of the Athens EQ. Indeed, the $\mathrm{kHz}$ EM activity associated with the preparation 


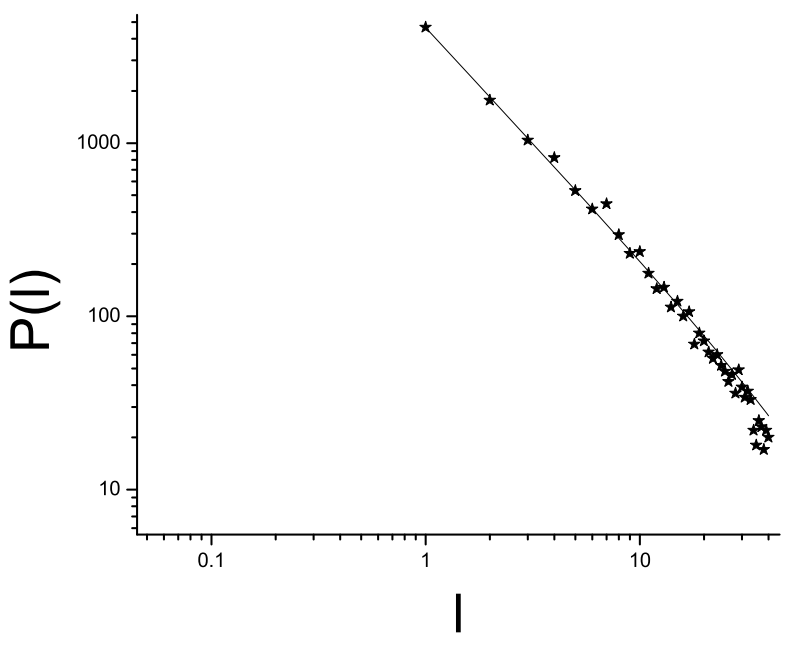

Fig. 12. A significant EQ with magnitude 6.9 occured in southern Greece on 14 February, 2008. Critical EM fluctuations have been recorded by the antenna finely tuned at $41 \mathrm{MHz}$ prior to this EQ (see text). The figure shows the distribution of the laminar lengths, as it produced by the analysis in terms of criticality, for the stationary window of critical fluctuations of duration approximately $36 \mathrm{~h}$. The fitting function (Eq. 4) is plotted by the solid line. The associated critical exponents are $p_{2}=1.32$ and $p_{3}=0.0072$. Importantly, the distribution of the laminar lengths of the critical fluctuations detected prior to the Athens EQ is characterized by the same $p_{2}, p_{3}$ exponents, namely, $p_{2}=1.30$ and $p_{3}=0.0077$.

of the Athens EQ is consistent with other precursors that are imposed by data from other disciplines such as: (i) seismology in terms of cumulative Benioff "strain", (ii) infrared remote sensing, (iii) synthetic aperture radars interferometry, and (iv) ultra low-frequency seismic electric signals (SES) (Karamanos et al., 2006). Notice, these measurements could indicate the possible position of the epicenter and the magnitude of the impending EQ, while the analysis of $\mathrm{MHz}-\mathrm{KHz} \mathrm{EM}$ emissions particularly point to way of estimating the time to global failure. The case of the Athens EQ suggests that through integrated search for the EQ precursors and of related physical mechanisms, we hope to lay foundation for EQ generation and thus to achieve a better short-term earthquake predictions.

Edited by: A. Tsonis

Reviewed by: A. Chmel

\section{References}

Alemany, P. and Zanette, D.: Fractal random walks from a variational formalism for Tsallis entropies, Phys. Rev. E, 49, R956R958, 1994.
Antoniou, N., Contoyiannis, Y., Diakonos, F., Karanikas, A., and Ktorides, C.: Pion production from a critical QCD phase, Nucl. Phys. A, 693, 799-824, 2001.

Baldovin, F. and Robledo, A.: Sensitivity to initial conditions at bifurcations in one-dimensional nonlinear maps: Rigorous nonextensive solutions, Europhys. Lett., 60, 518-524, 2002.

Buiatti, M., Grigolini, P., and Montagnini, A.: Dynamic Approach to the Thermodynamics of Superdiffusion, Phys. Rev. Lett., 82, 3383-3387, 1999.

Contoyiannis, Y. and Diakonos, F.: Criticality and imtermittency in the order parameter space, Phys. Lett. A, 268, 286-292, 2000.

Contoyiannis, Y., Diakonos, F., and Malakis, A.: Intermittent dynamics of critical fluctuations, Phys. Rev. Lett., 89, 035701/1-4, 2002.

Contoyiannis, Y., Kapiris, P., and Eftaxias, K.: Monitoring of a preseismic phase from its electromagnetic precursors, Phys. Rev. E, 71, 066123/1-14, 2005.

Contoyiannis, Y., Diakonos, F., Papaefthimiou, G., and Theophilidis, G.: Criticality in the relaxation phase of the spontaneous contracting atria isolated from the heart of the frog (Rana ridibunda), Phys. Rev. Lett., 93, 098101/1-4, 2004.

Contoyiannis, Y. and Diakonos, F.: Abrupt transition in a sandpile model, Phys. Rev. E, 73, 031303/1-5, 2006.

Diakonos, F., Pingel, D., and Schmelcher, P.: A stochastic approach to the construction of full-chaotic maps with prescribed statistical properties, Phys. Lett. A, 264, 162-170, 1999.

Kapiris, P., Eftaxias, K., and Chelidze, T.: The electromagnetic signature of prefecture criticality in heterogeneous media, Phys. Rev. Lett., 92, 065702/1-4, 2004.

Karamanos, K., Dakopoulos, D., Aloupis, K., Peratzakis, A., Athanasopoulou, L., Nikolopoulos, S., Kapiris, P., and Eftaxias, K.: Study of pre-seismic electromagnetic signals in terms of complexity, Phys. Rev. E, 74, 016104/1-21, 2006.

Karamanos, K., Peratzakis, A., Kapiris, P., Nikolopoulos, S., Kopanas, J., and Eftaxias, K.: Extracting pre-seismic electromagnetic signatures in terms of symbolic dynamics, Nonlin. Processes Geophys., 12, 835-848, 2005, http://www.nonlin-processes-geophys.net/12/835/2005/.

Koponen, I.: Analytic approach to the problem of convergence of truncated Levy flights towards the Gaussian stochastic process, Phys. Rev. E, 52, 1197-1199, 1995.

Mantegna, R. and Stanley, H.: Stochastic Process with Ultraslow Convergence to a Gaussian: The Truncated Levy Flights, Phys. Rev. Lett., 73, 2946-2949, 1994.

Pingel, D., Schmelcher, P., and Diakonos, F.: Theory and examples to the inverse Frobenious-Perron problem for complete chaotic maps, Chaos, 9, 357-366, 1999.

Robledo, A.: Critical fluctuations, Intermittent dynamics and Tsallis statistics, Physica A, 344, 631-636, 2004.

Scafetta, N. and West, B.: Multiscaling Comparative Analysis of Time Series and Geophysical Phenomena, Complexity, 10, 51$56,2005$.

Schuster, H.: Deterministic Chaos. An Introduction, VCH, 1989.

Tsallis, C.: Possible generalization of Boltzmann-Gibbs statistics, J. Stat. Phys., 52 479-487, 1988.

Vilar, C., Franca, G., Silva, R., and Alcaniz, J.: Nonextensivity in geological faults?, Physica A, 377, 285-290, 2007. 